\title{
Chlorococcales de la Amazonia Colombiana. II
}

\author{
RICARDO O. ECHENIQUE ${ }^{1}$, MARCELA NÚÑEZ-AVELLANEDA² y SANTIAGO R. DUQUE ${ }^{3}$
}

\begin{abstract}
Summary: Chlorococcales of the Colombian Amazon. II. In this paper 41 taxa belonging to the families Coelastraceae, Chlorococcaceae, Radiococcaceae, Palmellaceae, Oocystaceae, Characiaceae, Treubariaceae, Botryococcaceae, Micractiniaceae and Hydrodictyaceae of the order Chlorococcales were recorded. The material studied was collected in rivers and lakes from Colombian Amazon basin. Among the total taxa recorded, 20 are new for Colombia and 10 are new for the Colombian region of the basin. The family best represented in terms of species richness was Oocystaceae, with 10 taxa, and the largest number of taxa was found in lakes Yahuarcaca (Amazon River).
\end{abstract}

Key words: Colombian Amazon basin, Chlorococcales, taxonomy.

\begin{abstract}
Resumen: En el presente trabajo se registran 41 taxa, pertenecientes a las familias Coelastraceae, Chlorococcaceae, Radiococcaceae, Palmellaceae, Oocystaceae, Characiaceae, Treubariaceae, Botryococcaceae, Micractiniaceae e Hydrodictyaceae (orden Chlrorococcales) hallados en ríos y lagos de la cuenca de Amazonia Colombiana. Del total encontrado, 20 son nuevas citas para el país y 10 para la cuenca en Colombia. La familia mejor representada, en cuanto a riqueza específica, fue Oocystaceae con 10 taxa y en los lagos Yahuarcaca (Río Amazonas) se ha registrado el mayor número de taxa del orden.
\end{abstract}

Palabras clave: Amazonia-colombiana, Chlorococcales, taxonomía.

\section{INTRODUCCIÓN}

Estudios limnológicos en Colombia han permitido conocer las condiciones ambientales básicas de varios ríos y lagos, en especial de la cuenca del Amazonas (Solimões), Putumayo (Içã) y Caquetá o Japurá (Duque et al., 1997; NúñezAvellaneda \& Duque, 2001).

Una de las taxocenosis más estudiada es la de las microalgas, como se demuestra en Duque \& Núñez-Avellaneda (2000). La ficoflora en la región ha sido inventariada en los grupos Euglenophycae,

1 Facultad de Ciencias Naturales y Museo (UNLP) y CIC (Bs. As.). Paseo del Bosque s/n, 1900, La Plata. Argentina.rechen@fcnym.unlp.edu.ar

2 Instituto Amazónico de Investigaciones Científicas, Sinchi. Vía Aeropuerto, Leticia (Amazonas), Colombia. lunamarcela@hotmail.com

3 Instituto Amazónico de Investigaciones, Imani. Universidad Nacional de Colombia - Sede Leticia. A. A. \# 215, Leticia (Amazonas), Colombia. srduquee@ bt.unal.edu.co
Chlorophyceae, Zygophyceae, Bacillariophyceae y Chrysophyceae. En algas verdes y particularmente en el orden Chlorococcales tenemos los trabajos de Núñez-Avellaneda \& Duque (1998) y Echenique et al. (2003). En estas contribuciones se registran para el orden 51 taxa en la Amazonia colombiana.

En el presente trabajo se continúa con el inventario de este orden, con registros que en este caso pertenecen a las familias Coelastraceae, Chlorococcaceae, Radiococcaceae, Palmellaceae, Oocystaceae, Characiaceae, Treubariaceae, Botryococcaceae, Micractiniaceae e Hydrodictyaceae.

\section{Materiales y Métodos}

Se analizó un total de 18 muestras pertenecientes a 11 lugares situados entre lagos y ríos de las tres cuencas visitadas, Amazonas, Putumayo y Caquetá (Fig. 1). Para mayor detalle de los sitios de muestreo ver Duque et al. (1997). En la Tabla 1 


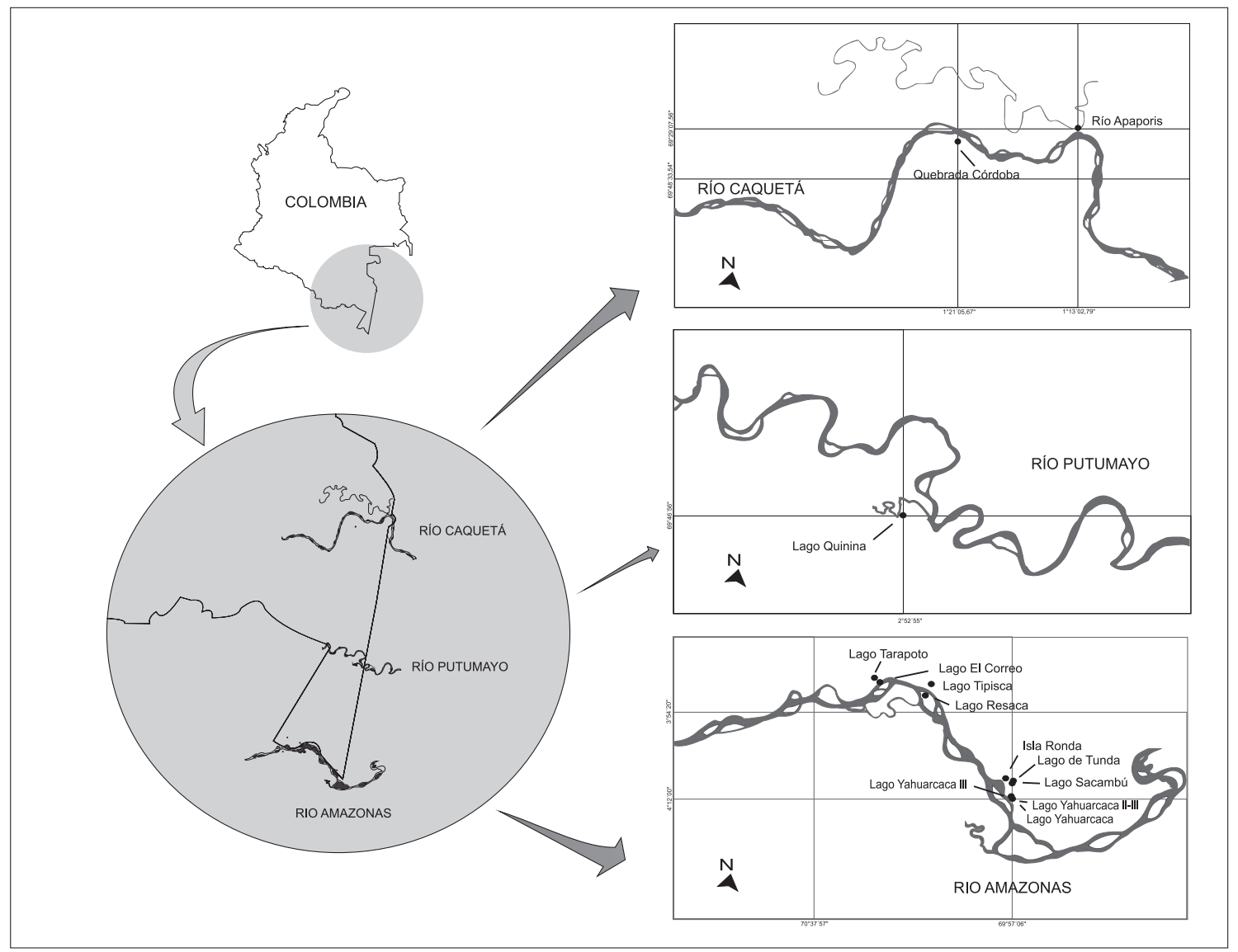

Fig. 1. Área de estudio y ubicación de las estaciones de muestreo.

se indican los sitios y tipos de ambientes visitados; en un solo caso se registra un agua intermedia definida así por Duque et al. (1997) para sistemas pobres en electrolitos pero alta carga de material en suspensión.

Las muestras colectadas en las tres cuencas corresponden a material planctónico (ticoplancton y eufitoplancton); la primera taxocenosis obtenida por estrujamiento de macrófitas y la segunda por arrastres de redes de plancton de mallas de 11, 17 y $24 \mu \mathrm{m}$ de poro. Todas las muestras fueron fijadas con solución de Transeau (Prescott, 1961).

El estudio taxonómico fue realizado utilizando microscopios ópticos Olympus y Wild M20, provistos de cámara de dibujo y ocular para medición. En las figuras, la escala gráfica equivale a $10 \mu \mathrm{m}$. Para la identificación y clasificación de los diferentes taxa se utilizaron, principalmente, los trabajos de Komárek (1983), Komárek \&
Fott (1983), Hindák (1977, 1984, 1988, 1990) y Comas (1996). Para determinar la presencia de los taxa en Colombia, se revisó una base de datos del Instituto Amazónico de Investigaciones (Imani) de la Universidad Nacional de Colombia y para la cuenca amazónico-colombiana los trabajos de Duque \& Núñez-Avellaneda (2000) y Echenique et al. (2003). Todo el material se encuentra depositado en la Colección "Ficoteca Amazónica" de la Universidad Nacional de Colombia - Sede Leticia.

\section{Resultados}

Se presenta a continuación una breve descripción, sitio de observación y datos de distribución en Colombia y en la cuenca, de los 41 taxa encontrados y su registro para Colombia y la cuenca amazónica dentro del país. 
Tabla 1. Número de herbario, localidad, hábito (T: ticoplancton; F: fitoplancton) y fecha de colecta de las muestras analizadas.

\begin{tabular}{|c|c|c|c|c|}
\hline Muestra & Localidad & Coordenadas geográficas & Hábito & Fecha \\
\hline 0022 & Lago Isla Ronda, Río Amazonas. & $4^{\circ} 09^{\prime} 00^{\prime \prime} \mathrm{S}-69^{\circ} 57^{\prime} 22^{\prime \prime} \mathrm{W}$ & $\mathrm{T}$ & $12 / 1 / 1990$ \\
\hline 0054 & Lago Tarapoto, Río Loreto Yacu & $3^{\circ} 47^{\prime} 18^{\prime \prime} \mathrm{S}-70^{\circ} 25^{\prime} 30^{\prime \prime} \mathrm{W}$ & $\mathrm{F}$ & $6 / 29 / 1991$ \\
\hline 0057 & Lago Yahuarcaca, Río Amazonas & $4^{\circ} 12^{\prime} 00^{\prime \prime} \mathrm{S}-69^{\circ} 57^{\prime} 06^{\prime \prime} \mathrm{W}$ & $\mathrm{F}$ & $7 / 12 / 1991$ \\
\hline 0129 & Lago Tipisca, Río Amacayacu & $3^{\circ} 47^{\prime} 18^{\prime \prime} \mathrm{S}-70^{\circ} 18^{\prime} 21^{\prime \prime} \mathrm{W}$ & $\mathrm{F}$ & $7 / 24 / 1991$ \\
\hline 0130 & Lago Resaca, Río Amazonas. & $3^{\circ} 51^{\prime} 00^{\prime \prime} \mathrm{S}-70^{\circ} 15^{\prime} 35^{\prime \prime} \mathrm{W}$ & $\mathrm{F}$ & $5 / 25 / 1991$ \\
\hline 0196 & Lago Quinina, Río Putumayo & $2^{\circ} 52^{\prime} 55^{\prime \prime} \mathrm{S}-69^{\circ} 46^{\prime} 56^{\prime \prime} \mathrm{W}$ & $\mathrm{F}$ & $7 / 31 / 1994$ \\
\hline 0242 & Lago Sacambú, Río Amazonas & $4^{\circ} 10^{\prime} 35^{\prime \prime} \mathrm{S}-69^{\circ} 58^{\prime} 31^{\prime \prime} \mathrm{W}$ & $\mathrm{F}$ & 8/10/1994 \\
\hline 0250 & Lago Tarapoto, Río Amazonas & $3^{\circ} 47^{\prime} 18^{\prime \prime} \mathrm{S}-70^{\circ} 25^{\prime} 30^{\prime \prime} \mathrm{W}$ & $\mathrm{F}$ & 8/13/1994 \\
\hline 0257 & Lago El Correo, Río Amazonas & $3^{\circ} 48^{\prime} 03^{\prime \prime} \mathrm{S}-70^{\circ} 24^{\prime} 11^{\prime \prime} \mathrm{W}$ & $\mathrm{F}$ & 8/14/1994 \\
\hline 0272 & Lago Yahuarcaca III, Río Amazonas & $4^{\circ} 11^{\prime} 15^{\prime \prime} \mathrm{S}-69^{\circ} 57^{\prime} 22^{\prime \prime} \mathrm{W}$ & $\mathrm{F}$ & 8/16/1994 \\
\hline 0285 & Lago de Tunda, Río Amazonas. & $4^{\circ} 09^{\prime} 07^{\prime \prime} \mathrm{S}-69^{\circ} 57^{\prime} 50^{\prime \prime} \mathrm{W}$ & $\mathrm{F}$ & 8/16/1994 \\
\hline 0286 & Lago Yahuarcaca, Río Amazonas & $4^{\circ} 12^{\prime} 00^{\prime \prime} \mathrm{S}-69^{\circ} 57^{\prime} 06^{\prime \prime} \mathrm{W}$ & $\mathrm{F}$ & 8/16/1994 \\
\hline 0292 & Lago Yahuarcaca, Río Amazonas & $4^{\circ} 12^{\prime} 00^{\prime \prime} \mathrm{S}-69^{\circ} 57^{\prime} 06^{\prime \prime} \mathrm{W}$ & $\mathrm{F}$ & $11 / 3 / 1994$ \\
\hline 0295 & Lago Yahuarcaca, Río Amazonas & $4^{\circ} 12^{\prime} 00^{\prime \prime} \mathrm{S}-69^{\circ} 57^{\prime} 06^{\prime \prime} \mathrm{W}$ & $\mathrm{F}$ & $11 / 3 / 1994$ \\
\hline 0300 & Lago Yahuarcaca III, Río Amazonas & $4^{\circ} 11^{\prime} 15^{\prime \prime} \mathrm{S}-69^{\circ} 57^{\prime} 22^{\prime \prime} \mathrm{W}$ & $\mathrm{F}$ & $11 / 3 / 1994$ \\
\hline 0314 & Quebrada Córdoba, Río Caquetá & $1^{\circ} 21^{\prime} 05.67^{\prime \prime} \mathrm{S}-69^{\circ} 48^{\prime} 33.54^{\prime \prime} \mathrm{W}$ & $\mathrm{F}$ & $11 / 13 / 1994$ \\
\hline 0328 & Río Apaporis, Río Caquetá. & $1^{\circ} 13^{\prime} 02.79^{\prime \prime} \mathrm{S}-69^{\circ} 29^{\prime} 07.56^{\prime \prime} \mathrm{W}$ & $\mathrm{F}$ & 11/9/1994 \\
\hline 0378 & Lago Yahuarcaca II-III, Río Amazonas & $4^{\circ} 11^{\prime} 38^{\prime \prime} \mathrm{S}-69^{\circ} 57^{\prime} 24^{\prime \prime} \mathrm{W}$ & $\mathrm{F}$ & $7 / 2 / 1995$ \\
\hline
\end{tabular}

\section{Familia Coelastraceae}

\section{Género Actinastrum}

Actinastrum hantzschii var. hantzschii Lagerh. (Fig. 2A)

Cenobio compuesto por 4-8 células subcilindricas con el extremo interior redondeadoaplanado y el libre cónico-redondeado. Células dispuestas radialmente. Cloroplasto parietal con un pirenoide.

Dimensiones: L: $15-20 \mu \mathrm{m}$; A: 3-3,5 $\mu \mathrm{m}$.

Material estudiado: 0250, 0257, 0272, 0286 y 0378 .

Actinastrum hantzschii Lagerh. var. subtile Wolosz. (Fig. 2B)

Cenobio radial compuesto por 4-8 células alargadas, que se atenúan en forma aguzada hacia el extremo libre, el extremo interno ligeramente aplanado. Cloroplasto parietal con un pirenoide.

Dimensiones: L: 18-22 $\mu \mathrm{m}$; A: 2-3,5 $\mu \mathrm{m}$.

Material estudiado: 0054, 0196, 0272 y 0286.

Observaciones: en la mayoría de los ejemplares analizados, el diámetro celular resultó mayor que el mencionado por Komárek (1983), Comas
(1996) y Echenique \& Arenas (1994). Nueva para Colombia.

\section{Género Coelastrum}

Coelastrum astroideum De Not. (Fig. 2C)

Cenobios esféricos de 8-16 células ovales, ligeramente cuneiformes y unidas entre sí directamente por sus paredes. Cloroplasto parietal con un pirenoide.

Dimensiones: diámetro celular: 8-10 $\mu \mathrm{m}$.

Material estudiado: 0257.

\section{Coelastrum microporum Näg. (Fig. 2D)}

Cenobio esférico compuesto por 8-16 células esféricas, unidas entre sí por proyecciones de la pared celular poco evidentes. Cloroplasto parietal con un pirenoide.

Dimensiones: diámetro celular: $6,5-9 \mu \mathrm{m}$.

Material estudiado: 0250, 0257, 0285 y 0286.

Observaciones: nueva para la cuenca.

Coelastrum pulchrum Schmidle (Fig. 2E)

Cenobios esféricos formados por 8-32 células poliédricas unidas entre sí por medio de apéndices 

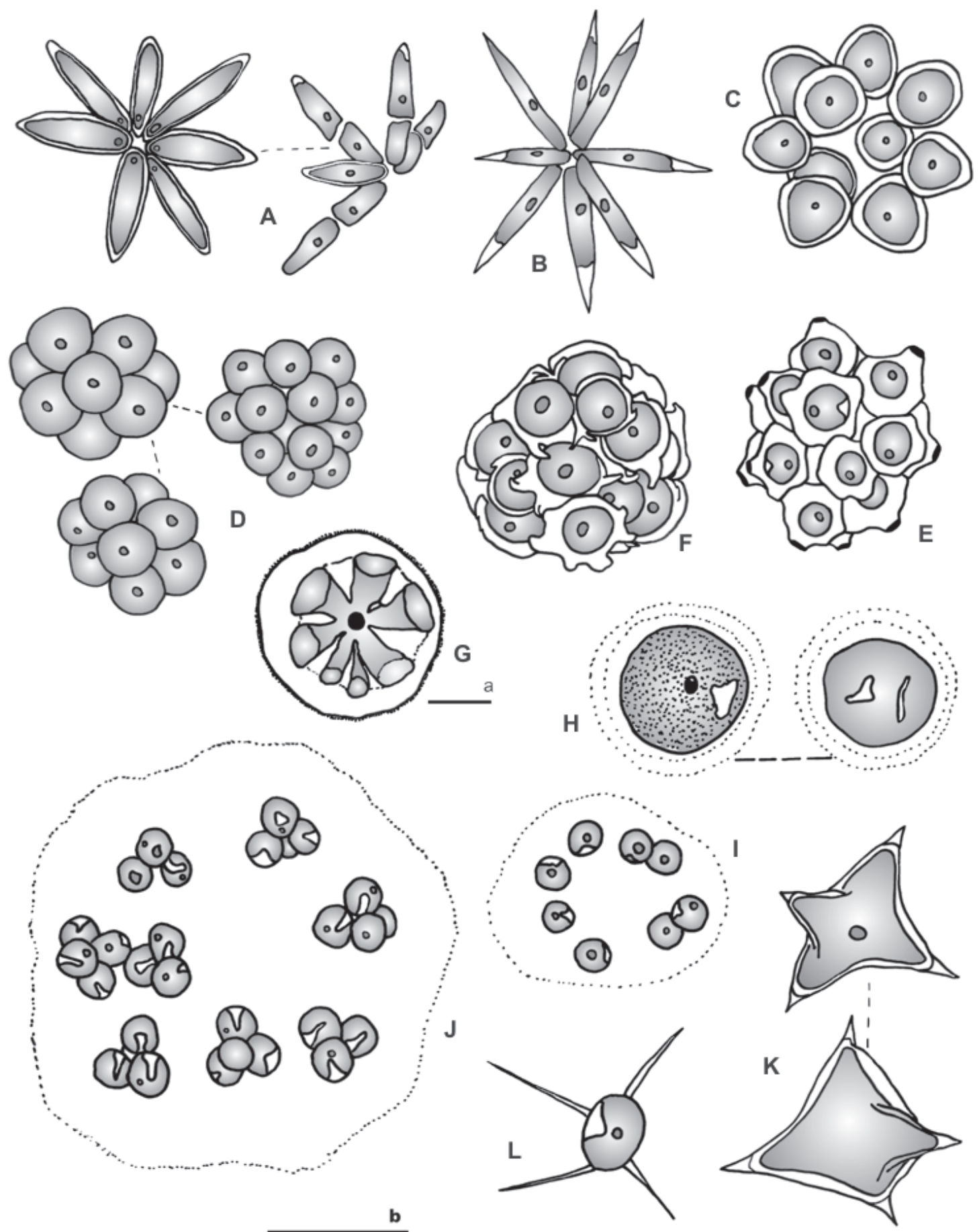

Fig. 2. A: Actinastrum hantzschii var. hantzschii. B: Actinastrum hantzschii var. subtile. C: Coelastrum astroideum. D: Coelastrum microporum. E: Coelastrum pulchrum. F: Coelastrum reticulatum. G: Actinochloris sphaerica. H: Neochloris pyrenoidosa. I: Coenoccoccus fottii. J: Coenococcus planctonicus. K: Chlorotetraedron incus. L: Lagerheimia subsalsa. La escala gráfica en la Fig. G está representada por la barra a, en el resto por la barra $b$. 
cónico-truncados y con un apéndice cónicoredondeado, en el extremo libre de cada célula. Cloroplasto parietal con un pirenoide.

Dimensiones: diámetro celular: 7-9 $\mu \mathrm{m}$.

Material estudiado: 0292.

\section{Coelastrum reticulatum (Dangeard) Senn (Fig. 2F)}

Cenobios esféricos formados por 8-32 células esféricas provistas de proyecciones cilíndricoalargadas, por medio de las cuales se unen entre sí.

Dimensiones: diámetro celular: 7,5-8,5 $\mu \mathrm{m}$.

Material estudiado: 0285 y 0292.

\section{Familia Chlorococcaceae}

Género Actinochloris

Actinochloris sphaerica Kors. (Fig. 2G)

Células esféricas a ligeramente ovales. Cloroplasto radialmente estrellado, con un pirenoide central. Vaina evidente.

Dimensiones: diámetro celular: $37 \mu \mathrm{m}$.

Material estudiado: 0130.

Observaciones: nueva para la cuenca.

\section{Género Neochloris}

Neochloris pyrenoidosa Arce \& Bold (Fig. 2H)

Células esféricas; cloroplasto parietal que ocupa prácticamente todo el volumen celular, con un pirenoide.

Dimensiones: diámetro celular: $16-18 \mu \mathrm{m}$.

Material estudiado: 0257 y 0272.

Observaciones: nueva para Colombia.

\section{Familia Radiococcaceae}

Género Coenococcus

Coenoccoccus fottii Hind. (Fig. 2I)

Colonias globosas, gelatinosas, de (4)-8-(16) células esféricas que se alternan en dos niveles. Cloroplasto parietal con un pirenoide.

Dimensiones: 4,5-5 $\mu \mathrm{m}$.

Material estudiado: 0292.

Observaciones: Hindák (1977) describe a esta especie para poblaciones de Sphaerocystis schroeteri que solo se reproducen por autosporas (Fanés Treviño, 2008). Nueva para Colombia.

\section{Coenococcus planctonicus Kors. (Fig. 2J)}

Colonias gelatinosas, de borde irregular, conteniendo (16)-32-(64) células esféricas, agrupadas por lo general en tétradas, o bien en grupos de 8 células. Cloroplasto parietal con un pirenoide.

Dimensiones: diámetro celular: 5-6,5 $\mu \mathrm{m}$.

Material estudiado: 0292.

Observaciones: nueva para Colombia.

\section{Familia Palmelaceae}

\section{Género Chlorotetraedron}

Chlorotetraedron incus (Teil.) Kom. \& Kov. (Fig. 2K)

Células tetragonales de márgenes cóncavos, con ángulos agudos, de extremos redondeados que se prolongan en espinas gruesas y rectas. Cloroplasto parietal con un pirenoide.

Dimensiones: diámetro celular: 14-16 $\mu \mathrm{m}$; espinas: $4-4,5 \mu \mathrm{m}$.

Material estudiado: 0272 y 0286.

Observaciones: nueva para Colombia.

\section{Familia Oocystaceae}

Género Lagerheimia

Lagerheimia subsalsa Lemm. (Fig. 2L)

Células solitarias, elipsoidales, de polos redondeados, provistos de cuatro espinas. Cloroplasto parietal con un pirenoide.

Dimensiones: L: $11 \mu \mathrm{m}$; A: $8 \mu \mathrm{m}$; espinas: $11-15$ $\mu \mathrm{m}$.

Material estudiado: 0257.

Observaciones: en Colombia solo fue citada por Ramírez (1992) como Chodatella subsalsa.

\section{Género Granulocystopsis}

Granulocystopsis pseudocoronata (Kors.) Hind. (Fig. 3A)

Células ampliamente ovales con engrosamientos polares evidentes, reunidas formando pequeñas colonias. Cloroplasto parietal con un pirenoide.

Dimensiones: L: 18-21 $\mu \mathrm{m}$; A: 16-17,5 $\mu \mathrm{m}$.

Material estudiado: 0292.

Observaciones: nueva para Colombia.

\section{Género Nephrocytium}

Nephrocytium agardhianum Näg. (Fig. 3B)

Colonias elipsoidales gelatinosas. Células reniformes, ligeramente curvadas, dispuestas dentro de una envoltura mucilaginosa oviforme. Cloroplasto parietal con un pirenoide.

Dimensiones: L: 13-15 $\mu \mathrm{m}$; A: 5-6 $\mu \mathrm{m}$.

Material estudiado: 0300 y 0378.

Observaciones: nueva para la cuenca. 
Bol. Soc. Argent. Bot. 48 (3-4) 2013

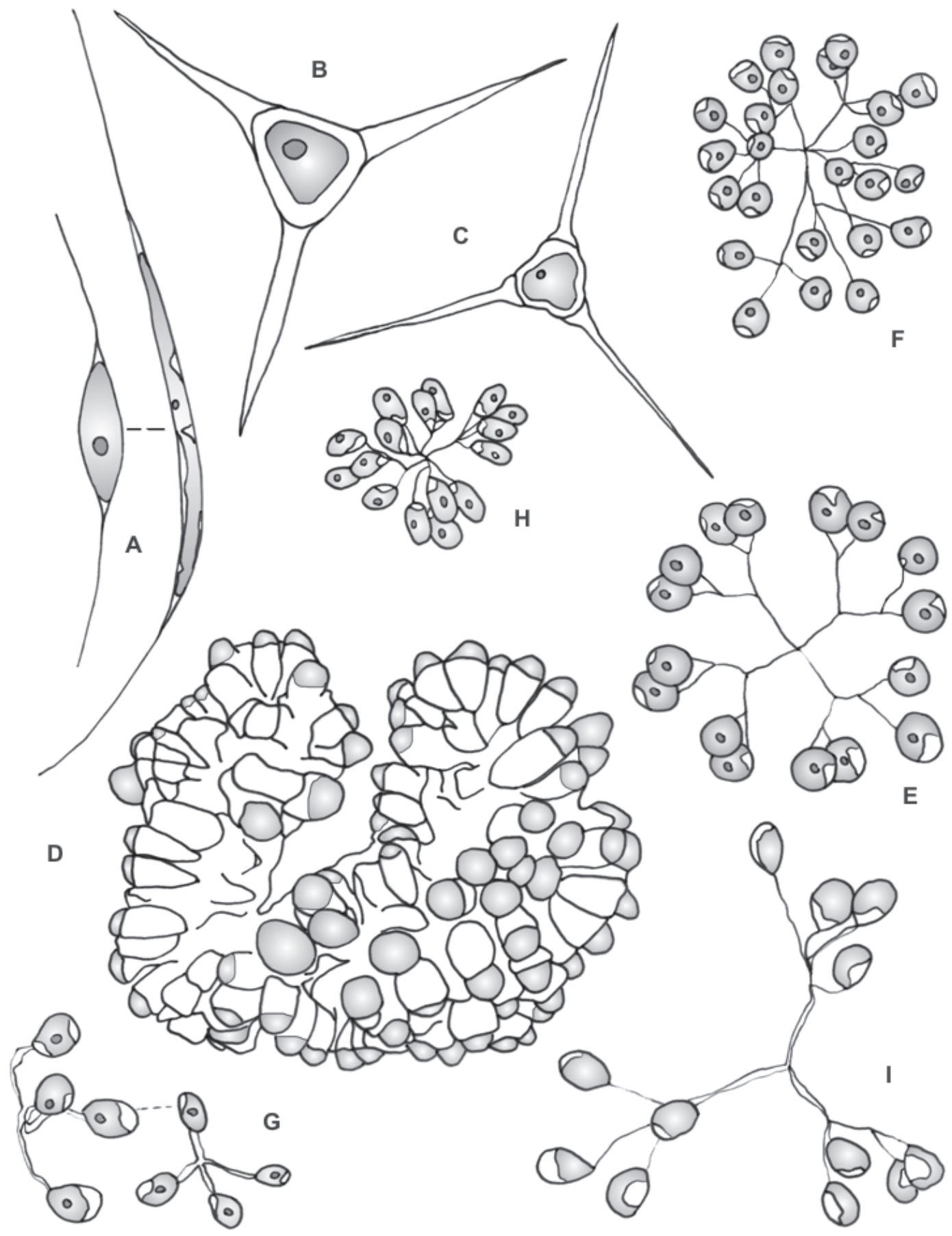

Fig. 3. A: Granulocystopsis pseudocoronata. B: Nephrocytium agardhianum. C: Nephrocytium allantoideum. D: Nephrocytium limneticum. E: Nephrocytium aff. schilleri. F: Oocystella solitaria. G: Oocystis elliptica. H: Oocystella lacustris. I: Siderocytopsis aff. irregularis. La escala gráfica equivale a $10 \mu \mathrm{m}$. 


\section{R. O. Echenique et al. - Chlorococcales de la Amazonia Colombiana}

Nephrocytium allantoideum Bohl. (Fig. 3C)

Colonias elipsoidales compuestas por 2-4 células cilíndricas a cilíndrico-fusiformes, algo curvadas, inmersas en una amplia y globosa envoltura mucilaginosa. Cloroplasto parietal con un pirenoide.

Dimensiones: L: 15-18 $\mu \mathrm{m}$; A: 3-7 $\mu \mathrm{m}$.

Material estudiado: 0022.

Observaciones: a diferencia de lo descripto por Komárek \& Fott (1983) y Comas (1996), en nuestro material se encontraron colonias de 2 células y en algunos ejemplares presentaron células algo más cortas y más anchas. Nueva para Colombia.

Nephrocytium limneticum (G.M. Smith) G.M. Smith (Fig. 3D)

Colonias ampliamente ovales, formadas por células cilíndrico-reniformes, muy poco curvadas, incluidas en una amplia vaina mucilaginosa. Cloroplasto parietal con un pirenoide.

Dimensiones: L: 14-24 $\mu \mathrm{m}$; A: 8-14 $\mu \mathrm{m}$.

Material estudiado: 0022 y 0292.

Observaciones: nueva para Colombia.

Nephrocytium aff. schilleri (Kamm.) Com. (Fig. 3E)

Colonias globosas compuestas por células semilunulares, con los extremos ligeramente aguzados, inmersos en una amplia vaina gelatinosa. Cloroplasto parietal.

Dimensiones: L: 15-18 $\mu \mathrm{m}$; A: 3-7 $\mu \mathrm{m}$.

Material estudiado: 0242.

Observaciones: no se observaron pirenoides.

\section{Género Oocystella}

Oocystella lacustris (Chod.) Hindak (Fig. 3H)

Células elíptico-ovales, de polos suavemente redondeados y sin engrosamientos. Cloroplasto parietal (1-2), con un pirenoide. Pared materna globosa.

Dimensiones: L: 11-12 $\mu \mathrm{m}$; A: 6,5-8 $\mu \mathrm{m}$.

Material estudiado: 0022 y 0285.

Observaciones: nueva para la cuenca.

Oocystella solitaria (Wittr. in Wittr. \& Nordst.) Hind. (Fig. 3F)

Células ovoides a citriformes, solitarias o agrupadas dentro de la pared materna, con engrosamientos polares. Cloroplastos numerosos, cada uno con un pirenoide.
Dimensiones: L: 29-31 $\mu \mathrm{m}$; A: 20-23 $\mu \mathrm{m}$.

Material estudiado: 0314.

Observaciones: nueva para la cuenca.

\section{Género Oocystis}

Oocystis elliptica W. West (Fig. 3G)

Células elipsoidales hasta subcilindricas, de polos ampliamente redondeados y sin engrosamientos. Numerosos cloroplastos globosos, sin pirenoide. Pared materna algo irregular.

Dimensiones: L: 14-16 $\mu \mathrm{m}$; A: 8-9 $\mu \mathrm{m}$.

Material estudiado: 0196.

Observaciones: nueva para la cuenca.

\section{Género Siderocystopsis}

Siderocytopsis aff. irregularis (Hind.) Hind. (Fig. 3I)

Células aproximadamente esféricas de las que se proyectan pequeñas "columnas", de las cuales surgen finas sedas. Vaina fina, hialina. Cloroplasto cupuliforme, con un pirenoide.

Dimensiones: diámetro celular: 8-10 $\mu \mathrm{m}$.

Material estudiado: 0054.

Observaciones: los ejemplares hallados en nuestro material son de tamaño mayor que los descriptos por Hindák (1988), (4-6 $\mu \mathrm{m}$ de diámetro celular). Nueva para Colombia.

\section{Familia Characiaceae}

\section{Género Schroederia}

Schroederia setigera (Schröd.) Lemm. (Fig. 4A)

Células alargado-fusiformes, rectas o ligeramente curvadas, con los polos prolongados en largas y finas espinas. Cloroplasto parietal con un pirenoide.

Dimensiones: L: 22,5-60 $\mu \mathrm{m}$; A: 2-6 $\mu \mathrm{m}$.

Material estudiado: 0196, 0257, 0272 y 0300.

Observaciones: nueva para la cuenca.

\section{Familia Treubariaceae}

\section{Género Treubaria}

Treubaria schmidlei (Schröd.) Fott y Kovac. (Fig. 4B)

Células triangulares con las paredes celulares convexas. Ángulos prolongados en largos y rectos apéndices hialinos espiniformes. Cloroplasto parietal con un pirenoide.

Dimensiones: diámetro celular: 15-17 $\mu \mathrm{m}$; apéndices: $25-30 \mu \mathrm{m}$.

Material estudiado: 0285, 0292, 0300 y 0378.

Observaciones: nueva para Colombia. 
Bol. Soc. Argent. Bot. 48 (3-4) 2013

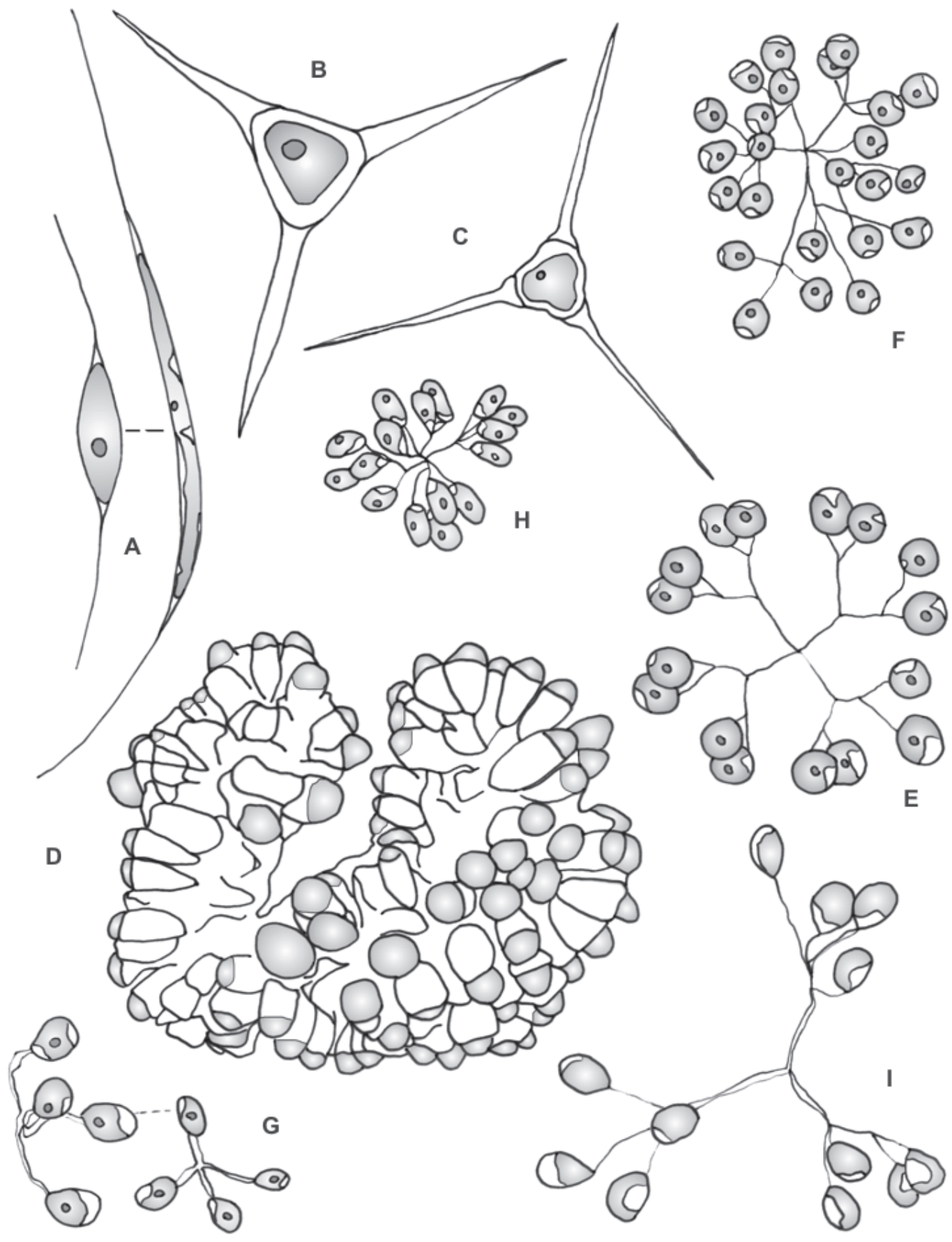

Fig. 4. A: Schroederia setigera. B: Treubaria schmidlei. C: Treubaria triappendiculata. D: Botryococcus braunii. E: Dictyosphaerium pulchellum var. pulchellum. F: Dictyosphaerium pulchellum var. minutum. G: Dictyosphaerium simplex. H: Dictyosphaerium tetrachotomum. I: Pseudodictyosphaerium anomalum. La escala gráfica equivale a $10 \mu \mathrm{m}$. 


\section{Treubaria triappendiculata Bern. (Fig. 4C)}

Células triangulares a tetragonales con las paredes celulares cóncavas. Ángulos prolongados en largos y rectos apéndices hialinos espiniformes. Cloroplasto parietal con un pirenoide.

Dimensiones: diámetro celular: 10-11 $\mu \mathrm{m}$; apéndices: $30-31 \mu \mathrm{m}$.

Material estudiado: 0054 y 0257.

Observaciones: nueva para Colombia.

\section{Familia Botryococcaceae}

\section{Género Botryococcus}

Botryococcus braunii Kütz. (Fig. 4D)

Colonias irregulares. Células ovales a oviformes dispuestas dentro de vainas individuales cupuliformes, en la periferia de una amplia matriz mucilaginosa. Cloroplasto parietal con un pirenoide.

Dimensiones: L: 11-16 $\mu \mathrm{m}$; A: 4,5-7 $\mu \mathrm{m}$.

Material estudiado: 0022 y 0292.

\section{Género Dictyosphaerium}

Dictyosphaerium pulchellum var. pulchellum Wood (Fig. 4E)

Colonias esféricas a ovoides. Células esféricas, dispuestas en el extremo de finos y largos pedicelos hialinos. Cloroplasto parietal, acopado, con un pirenoide.

Dimensiones: diámetro celular: 5,5-8 $\mu \mathrm{m}$.

Material estudiado: 0054, 0196, 0242, 0257, 0272, 0286, 0292, 0328 y 0378.

Dictyosphaerium pulchellum Wood var. minutum Defl. (Fig. 4F)

Células aproximadamente esféricas de pequeño tamaño, situadas en el extremo de finos y largos pedicelos hialinos, formando colonias esféricas a elípticas. Cloroplasto parietal, acopado, con un pirenoide.

Dimensiones: diámetro celular: 3,5-4 $\mu \mathrm{m}$.

Material estudiado: 0022.

Observaciones: nueva para Colombia.

\section{Dictyosphaerium simplex Kors. (Fig. 4G)}

Colonias pequeñas, de 2-4, muy raramente más células elípticas, ubicadas en los extremos de pedicelos hialinos, más bien cortos. Cloroplasto parietal con un pirenoide.

Dimensiones: L: 4,5-6 $\mu \mathrm{m}$; A: 3,5-4 $\mu \mathrm{m}$.

Material estudiado: 0022.

Observaciones: esta especie fue descripta por
Komárek \& Permann (1978) como D. chlorelloides. Hindák (1988) redefine a este taxón como $D$. simplex. Nueva para la cuenca.

Dictyosphaerium tetrachotomum Printz (Fig. 4H)

Colonias irregulares a esféricas, e incluso elípticas. Células ovales a elípticas, situadas, a partir del eje longitudinal de cada célula, en el extremo de finos pedicelos hialinos. Cloroplasto parietal acopado con un pirenoide.

Dimensiones: L: 6-7,5 $\mu \mathrm{m}$; A: 3,5-4,5 $\mu \mathrm{m}$.

Material estudiado: 0272.

Observaciones: nueva para Colombia.

\section{Género Pseudodictyosphaerium}

Pseudodictyosphaerium anomalum (Kors.) Hind. (Figs. 4I y 5A)

Colonias aproximadamente esféricas. Células oval-elípticas a ovoides, ubicadas en el extremo de delgados y prolongados pedicelos hialinos. Cloroplasto parietal sin pirenoide.

Dimensiones: L: 6-7 $\mu \mathrm{m}$; A: 4,5-5,5 $\mu \mathrm{m}$.

Material estudiado: 0196 y 0257.

Observaciones: nueva para Colombia.

\section{Familia Micractiniaceae \\ Género Micractinium \\ Micractinium bornhemiense (Corn.) Kors. (Fig. 5B)}

Colonias tetraédricas, con grupos de células reunidos en forma piramidal, en cada uno de los vértices. Células esféricas provistas de finas y largas sedas. Cloroplasto parietal.

Dimensiones: diámetro celular: 3-4 $\mu \mathrm{m}$; espinas: 16-30 $\mu \mathrm{m}$.

Material estudiado: 0242 y 0285.

Observaciones: en nuestro material no pudo observarse la presencia de pirenoides y las espinas son más cortas que las descriptas en Komárek \& Fott (1983). Nueva para Colombia.

Micractinium pusillum Fres. (Fig. 5C)

Colonias subesféricas. Células esféricas, provistas de largas y finas sedas. Cloroplasto parietal con un pirenoide.

Dimensiones: diámetro celular: 4,5-5,5 $\mu \mathrm{m}$; espinas: $17-25 \mu \mathrm{m}$.

Material estudiado: 0257, 0272, 0286, 0292 y 0378 .

Observaciones: nueva para Colombia. 
Bol. Soc. Argent. Bot. 48 (3-4) 2013
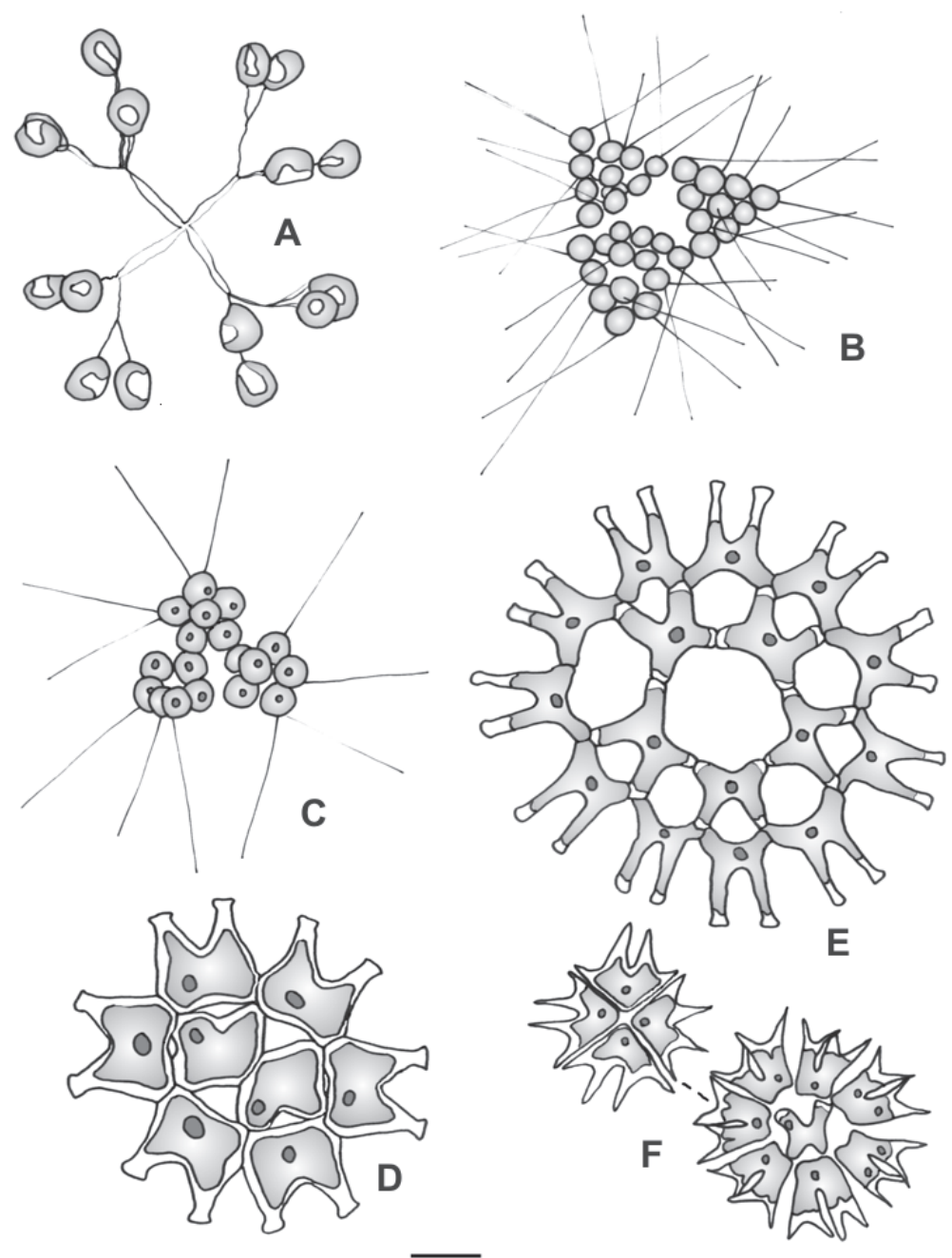

Fig. 5. A: Pseudodictyosphaerium anomalum. B: Micractinium bornhemiense. C: Micractinium pusillum. D: Pediastrum duplex var. duplex. E: Lacunastrum gracillimum. F: Parapediastrum longicornutum. La escala gráfica equivale a $10 \mu \mathrm{m}$.

\section{Familia Hydrodictiaceae}

\section{Género Lacunastrum}

Lacunastrum gracillimum (W. \& G.S. West) H. McManus (Fig. 5E)

Cenobio plano clatrado. Células con forma de $\mathrm{H}$, formando series concéntricas. Cuerpo celular central, reducido, del cual se proyectan dos procesos aproximadamente cilíndricos, hacia el exterior del cenobio, largos y hacia el interior algo más cortos.

Dimensiones: L: $13-15 \mu \mathrm{m}$; A: $10-13 \mu \mathrm{m}$.

Material estudiado: 0292, 0300 y 0378.

Observaciones: nueva para la cuenca. Este género y variedad se distinguen de Pediastrum duplex Meyen a partir de diferencias en el largo de los procesos y la ultraestructura de la pared celular (McManus et al., 2011).

\section{Género Monactinus}

Monactinus simplex var. simplex (Meyen) Corda (Fig. 6A)

Cenobio plano clatrado. Células, aproximadamente trapezoidales formando series concéntricas, las del anillo marginal con el extremo distal aproximadamente cilíndrico- 


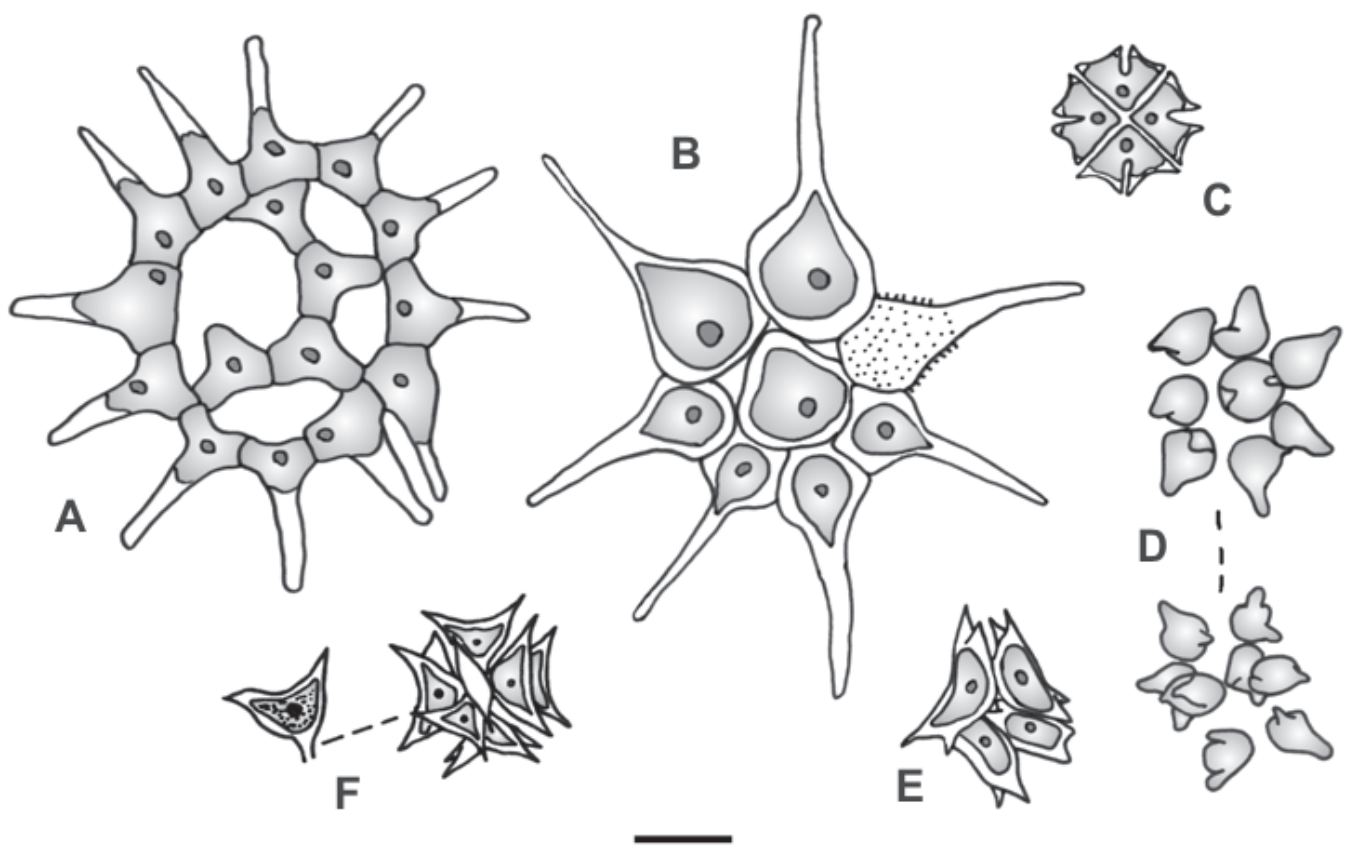

Fig. 6. A: Monactinus simplex var. simplex. B: Monactinus simplex var. echinulatum. C: Stauridium tetras. D: Sorastrum bengalicum. E: Sorastrum indicum. F: Sorastrum spinulosum. La escala gráfica equivale a $10 \mu \mathrm{m}$.

alargado. Pared celular muy finamente punteada. Cloroplasto parietal con un pirenoide.

Dimensiones: L: 13-16 $\mu \mathrm{m}$; A: 7-9 $\mu \mathrm{m}$.

Material estudiado: 0057 y 0285.

Observaciones: especie transferida por Buchheim et al. (2005).

Monactinus simplex (Meyen) Corda var. echinulatum (Wittr.) Pérez, Maidana \& Comas (Fig. 6B).

Cenobio plano no clatrado. Células globosas, con el extremo distal aproximadamente cilindricoalargado, formando series concéntricas. Pared irregularmente ornamentada densamente con pequeñas proyecciones espiniformes de hasta $1 \mu \mathrm{m}$ de largo. Cloroplasto parietal con un pirenoide.

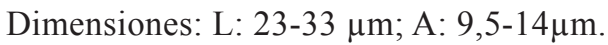

Material estudiado: 0285.

Observaciones: especie transferida por Pérez et al. (2009). Nueva para Colombia.

\section{Género Parapediastrum}

Parapediastrum longicornutum (Gutw.) Hegewald (Fig. 5F)

Cenobio plano clatrado. Células formando series, la central con forma de $\mathrm{H}$ y las de la periferia algo cuadrangulares con una fuerte incisión central en la cara externa, dando origen a dos amplios brazos que se bifurcan terminando, cada extremo, en una fuerte proyección espiniforme. Cloroplasto parietal con un pirenoide.

Dimensiones: L: 10-13 $\mu \mathrm{m}$; A: 9-15 $\mu \mathrm{m}$.

Material estudiado: 0054, 0129, 0257 y 0292.

Observaciones: especie transferida desde Pediastrum longicornutum (Gutw) Comas, por Buchheim et al. (2005). Nueva para Colombia.

\section{Género Pediastrum}

Pediastrum duplex var. duplex Meyen (Fig. 5D)

Cenobio plano clatrado. Células con forma de $\mathrm{H}$, con el cuerpo celular amplio, formando series concéntricas; las de la serie marginal con gruesos procesos corniformes de extremos truncados. Pared celular muy finamente punteada. Cloroplasto parietal con un pirenoide. 
Dimensiones: diámetro celular: 12,5-15 $\mu \mathrm{m}$. Material estudiado: 0285 y 0295.

\section{Género Stauridium}

Stauridium tetras (Ehr.) Hegewald (Fig. 6C)

Cenobio plano no clatrado. Células poligonales, formando series concéntricas, con una marcada incisión en la pared externa, aún en las de la serie interna. Las de la serie marginal, hacia el extremo distal terminan en cortos procesos espiniformes. Cloroplasto con un pirenoide.

Dimensiones: L: 6,5-12 $\mu \mathrm{m}$; A: 8,5-13 $\mu \mathrm{m}$.

Material estudiado: 0022, 0054, 0250, 0257, 0272 y 0286 .

Observaciones: especie transferida por Buchheim et al. (2005).

\section{Género Sorastrum}

Sorastrum bengalicum Philip (Fig. 6D)

Cenobio pequeño de 8 (32) células aproximadamente globosas, con dos pequeñas proyecciones subcilíndricas dispuestas en la parte externa de la pared celular. Cloroplasto parietal.

Dimensiones: L: 6,5-12 $\mu \mathrm{m}$; A: 8,5-13 $\mu \mathrm{m}$.

Material estudiado: 0272.

Observaciones: los cenobios estaban compuestos por un menor número de células, que los descriptos por Komárek \& Fott (1983) y las células se observaron algo desunidas entre sí. No se observa ningún elemento de conexión entre las células, ni pirenoides. Nueva para la cuenca.

\section{Sorastrum indicum Bern. (Fig. 6E)}

Cenobios globosos de 4 (8) células, aproximadamente, semilunares con los ángulos agudos; pared celular externa cóncava y la interna convexa. Cloroplasto parietal con un pirenoide.

Dimensiones: L: 6,5-7 $\mu \mathrm{m}$; A: 10,5-12 $\mu \mathrm{m}$.

Material estudiado: 0286.

Observaciones: nueva para Colombia.

\section{Sorastrum spinulosum Näg. (Fig. 6F)}

Células algo reniformes, con 4 proyecciones en la pared externa y una en la cara interna a través de las que se unen formando un cenobio aproximadamente esférico. Cloroplasto parietal con un pirenoide.

Dimensiones: L: 6-7 $\mu \mathrm{m}$; A: 12-14 $\mu \mathrm{m}$.

Material estudiado: 0378.

Observaciones: nueva para Colombia.

\section{Discusión}

Los ambientes visitados en las cuencas del Putumayo y del Caquetá difieren de los ambientes estudiados de la cuenca del Amazonas. Para Duque et al. (1997) y Núñez-Avellaneda \& Duque (2001) en las dos cuencas más al norte, ríos Putumayo y Caquetá, las aguas son blancas tipo II ( $\mathrm{pH}$ de 6.6, conductividad $15 \mu \mathrm{S} . \mathrm{cm}^{-1}$, cationes midiendo $\mathrm{Na}, \mathrm{K}, \mathrm{Ca}$ y $\mathrm{Mg}$ de $0.19 \mu$ eq. $\left.\mathrm{l}^{-1}\right)$. En tanto, las aguas del río Amazonas son blancas del tipo I ( $\mathrm{pH}$ de 7.6, conductividad $160 \mu \mathrm{S} . \mathrm{cm}^{-1}$ y cationes de $1.48 \mu$ eq. $1^{-1}$ ). En ambos casos las aguas blancas deben su nombre a que son ríos que nacen en los Andes. Mientras que la mayoría de los ambientes presentes en la región provienen de la propia llanura amazónica, encontrando que en el sector del Putumayo y del Caquetá las aguas son negras tipo II ( $\mathrm{pH}$ de 5.0 a 6.0, conductividad 12- $20 \mu{\mathrm{S} . \mathrm{cm}^{-1}}^{\mathrm{y}}$ cationes de $0.14 \mu$ eq. $\mathrm{l}^{-1}$ ) y en el sector del Amazonas las aguas son negras tipo I ( $\mathrm{pH}$ de 6,0 - 7.1, conductividad $120-45 \mu{\mathrm{S} . \mathrm{cm}^{-1} \mathrm{y}}$ cationes de $0.82 \mu$ eq..$\left.^{-1}\right)$. En la Tabla 1 se indican los sitios y tipos de ambientes visitados. El lago Tipisca, cuenca del río Amazonas (0129, Tabla 1), corresponde a un ambiente catalogado como de aguas intermedias tipo I (Duque et al., 1997), pobre en electrolitos pero con mayor material en suspensión que las aguas negras circundantes tipo I. La diferencia estadísticamente significativa, según estos autores es que en el Amazonas hay mayor mineralización y $\mathrm{pH}$ y menor transparencia si comparamos con los ambientes de Putumayo y Caquetá. Además, esta mineralización también produce mayor desarrollo de macrófitas litorales y de tapetes flotantes en los lagos del Amazonas, muy pocos en el Putumayo e inexistentes en el Caquetá (Duque, 1997).

En virtud de los estudios realizados pudo hallarse un total de 41 taxa pertenecientes a las familias Coelastraceae (6), Chlorococcaceae (2), Radiococcaceae (2), Palmellaceae (1), Oocystaceae (10), Characiaceae (1), Treubariaceae (2), Botryococcaceae (6), Micractiniaceae (2) e Hydrodictiaceae (9), en la cuenca amazónico-colombiana. La familia mejor representada, en cuanto a riqueza específica, fue Oocystaceae. Del total de organismos hallados, 20 son nuevas citas para el país y corresponden a Actinastrum hantzschii var. 
subtile, Actinochloris sphaerica, A. sphaerica, Coenococcus planctonicus, Chlorotetraedron incus, Granulocystopsis pseudocoronata, Nephrocytium agardhianum, N. allantoideum, $N$. limneticum, Siderocytopsis aff. irregularis, Treubaria schmidlei, T. triappendiculata, Dictyosphaerium pulchellum var. minutum, D. tetrachotomum, Pseudodictyosphaerium anomalum, Micractinium bornhemiense, M. pusillum, Parapediastrum longicornutum, Monactinus simplex var. echinulatum y Sorastrum indicum. En tanto, para la región de la amazonia colombiana, se mencionan por primera vez 10 taxa: Coelastrum microporum, Actinochloris sphaerica, Granulocystopsis pseudocoronata, Oocystella solitaria, O. lacustris, Oocystis elliptica, Siderocytopsis aff. irregularis, Dictyosphaerium simplex, Lacunastrum. gracillimum y Sorastrum bengalicum.

Este trabajo junto con los precedentes de Núñez-Avellaneda \& Duque (1998) y Echenique et al. (2003), permiten ampliar el conocimiento de la flora de las Chlorococcales de la región a un total de 92 taxa.

La mayor riqueza del Orden Chlorococcales está relacionada con las características ambientales que les son favorables, entre ellas mayor mineralización y gran desarrollo de macrófitas litorales y tapetes flotantes, como existen en los lagos de Yahuarcaca donde obtenemos la mayor riqueza específica; de igual forma el grupo se presenta en otros lagos con las mismas condiciones como son las de Isla Ronda, El Correo y Tarapoto, todos de la cuenca del río Amazonas (Duque et al., 1997). Para el Putumayo observamos en el lago Quinina 5 taxa que también estarían relacionados con un efecto limitante generado por el sombreado favorecido por el desarrollo incipiente de macrófitas (Duque, 1997).

\section{Agradecimientos}

Los autores agradecen el apoyo financiero de la Universidad Nacional de La Plata (Argentina), de la Comisión de Investigaciones Científicas (Prov. de Buenos Aires, Argentina), del Instituto Amazónico de Investigaciones Científicas - SINCHI y de la Universidad Nacional de Colombia - Sede Leticia, así como de COLCIENCIAS (Colombia).

\section{Bibliografía}

BUCHHEIM, M., J. BUCHHEIM, T. CARLSON, A. BRABAND, D. HEPPERLE, L. KRIENITZ, M. WOLF \& E. HEGEWALD. 2005. Phylogeny of the Hydrodictyaceae (Chlorophyceae): inferences from rDNA data. J. Phycol. 41: 1039-1054.

COMAS, A. 1996. Las Chlorococcales dulceacuicolas de Cuba. Bibl. Phycol. 99. J. Cramer, Stuttgart.

DUQUE, S. R. 1997. Tipificación limnológica de algunos lagos de la Amazonia colombiana a través de la composición, biomasa y productividad del fitoplancton. Tesis Msc. Universidad Nacional de Colombia, Bogotá.

DUQUE, S. R., J. E. RUIZ, J. GÓMEZ \& E. ROESSLER. 1997. Limnología. En: IGAC (ed.). Zonificación ambiental para el plan modelo Colombo - Brasilero (Eje Apaporis - Tabatinga: PAT), pp. 71-134. Editorial Linotipia, Santafé de Bogotá.

DUQUE, S. R. \& M. NÚÑEZ-AVELLANEDA. 2000 Microalgas acuáticas de la Amazonía colombiana. Biota Colomb. 1: 208-216.

ECHENIQUE, R. O. \& P. M. ARENAS. 1994. Fitoplancton de la Laguna Vitel. I. Gayana Bot. 51: 89-104.

ECHENIQUE, R. O., M. NÚÑEZ-AVELLANEDA \& S. R. DUQUE. 2004. Chlorococcales de la Amazonia Colombiana I: Chlorellaceae y Scenedesmaceae. Caldasia 26: 37-51.

KOMÁREK, J. 1983. Contribution to the Chlorococcal algae of Cuba. Nova Hedwigia 37: 65-180.

KOMÁREK, J. \& B. FOTT. 1983. ChlorophyceaeChlorococcales. Das phytoplankton des Süsswassers, systematik u biologie. In: THIENEMMAN, A. (ed.), Die Binnenngewässer 16, 7. E. Schweizerbart'sche Verlagbuchhandlung, Stuttgart.

KOMÁREK, J. \& J. PERMAN. 1978. Review of the genus Dictyosphaerium (Chlorococcales). Arch. Hydrobiol. 51 (Algol. Stud. 10): 233-297.

HINDÁK, F. 1977. Studies on the chlorococcal algae (Chlorophyceae). I. Biol. Práce 23. VEDA, Bratislava.

HINDÁK, F. 1984. Studies on the chlorococcal algae (Chlorophyceae). III. Biol. Práce 30. VEDA, Bratislava.

HINDÁK, F. 1988. Studies on the chlorococcal algae (Chlorophyceae). IV. Biol. Práce 34. VEDA, Bratislava.

HINDÁK, F. 1990. Studies on the chlorococcal algae (Chlorophyceae). V. Biol. Práce 36, VEDA, Bratislava.

MCMANUS, H. A., L. A. LEWIS, \& E. T. SCHULTZ. 2011. Distinguishing multiple lineages of Pediastrum duplex with morphometrics and a proposal for Lacunastrum gen. nov. J. Phycol. 47: 123-130. 
Bol. Soc. Argent. Bot. 48 (3-4) 2013

NÚÑEZ-AVELLANEDA, M. \& S. R. DUQUE. 1998. Chlorococcales (algae: Chlorophyceae) found in aquatic environments of the Colombian amazon basin. Caldasia 20: 5-11.

NÚÑEZ-AVELLANEDA, M. \& S. R. DUQUE. 2001. Fitoplancton de algunos ríos y lagos de la Amazonia colombiana. In: FRANKY, C. \& C. ZARATE (eds.), Imani mundo: Estudios en la Amazonia colombiana, pp. 305-335. UNIBIBLOS, Bogotá.

PÉREZ, M. del C., N. I. MAIDANA \& A. COMAS. 2009. Phytoplankton composition of the Ebro River estuary, Spain. Acta Bot. Croat. 68: 11-27.
PRESCOTT, G. W. 1961. Algae of the western Great Lakes area with an illustrated key to the genera of desmids and freshwater diatoms. Revised edition. W.M.C. Brown Publishers, Dubuque, Iowa.

RAMÍREZ, J. J. 2000. Fitoplancton de agua dulce: Bases ecológicas, taxonómicas y sanitarias. Editorial Universidad de Antioquia, Medellín.

Recibido el 10 de agosto de 2012, aceptado el 15 de marzo de 2013. 